\title{
Simultaneous Detection of X-Ray Photoacoustic and Fluorescence Signals as Applied to Characterization of Layered Materials
}

\author{
Kenji Kato* and Yoshinori Sugitani** \\ *National Chemical Laboratory for Industry, Tsukuba, Ibaraki 305, Japan \\ **Department of Materials Science, Kanagawa University, Hiratsuka, Kanagawa 259-12, Japan
}

\begin{abstract}
A simultaneous measurement of photoacoustic and fluorescence signals has been attempted using an X-ray fluorescence spectrometer equipped with a photoacoustic cell designed for the present purpose. The combined measurement was conducted for metal foils and model samples with layered structures. The fluorescence measurement gave information concerning the elements of the samples and the photoacoustic signal gave information concerning the total amount of $\mathrm{X}$-ray absorption and the thermal properties of the samples. The same samples were also examined through ordinary photoacoustic measurements by visible light. The use of different excitation wavelengths in the combined measurement was found to give more detailed information about the thermal and structural properties than the individual use of these techniques.
\end{abstract}

Keywords Photoacoustic, X-ray fluorescence, simultaneous measurement, X-ray excitation, layered material characterization, scanning observation

Photoacoustic techniques have been widely used in studies concerning the thermal properties of bulk and layered materials through non-destructive observation. ${ }^{1}$ Recently, photoacoustic measurements in the X-ray region were successfully conducted using a conventional $\mathrm{X}$-ray tube ${ }^{2-4}$ and synchrotron radiation 5,6 as the exciting source. Synchrotron radiation (SR) is known to be an excellent X-ray source because of its strong intensity, wide selectivity of exciting wavelengths, and directional nature of the light beam. Particularly, elemental analysis using characteristic X-ray absorption of atoms is an established analytical tool. However, its difficulty lies in the fact that a synchrotron radiation facility is not easily accessed. On the other hand, X-ray photoacoustic measurements using an X-ray tube as the exciting source are easily conducted, though elemental analysis is not accurately conducted as in SR experiments. The X-ray fluorescence method is advantageous in terms of non-destructive elemental analysis as well as the convenience of operation, while the results of measurements are strongly dependent on the state of the specimen. Photoacoustic measurements using an $\mathrm{X}$-ray tube combined with X-ray fluorescence measurements will provide more detailed information concerning the amount of net absorption, the thermal and geometrical properties of layered materials. Furthermore, in the X-ray region, a comparison of the three wavelength regions is of interest, since the samples have different absorption properties from the ordinary UV and visible wavelength regions.
The simultaneous use of photoacoustic and fluorescent techniques provides a versatile analytical tool for materials science.

\section{Experimental}

A built-in type $\mathrm{X}$-ray tube with a molybdenum target was used as the exciting source. Figure 1 shows a schematic diagram of the experimental setup. The X-ray beam was narrowed and collimated by the molybdenum collimator with a diameter of $1 \mathrm{~mm}$. A molybdenum spinning rod with a through hole was used to chop the exciting beam. The body of the photoacoustic cell was made of brass, and windows and their frames were made of beryllium and aluminum, respectively. The thicknesses of the windows were $0.5 \mathrm{~mm}$ (upper) and $0.25 \mathrm{~mm}$ (lower). The sample room was designed so as to be close to the detector in order to avoid fluorescence and scattered X-rays from the cell wall. The cell was mounted on a sliding stage. X-Ray fluorescence was detected by a solid-state detector, $\mathrm{Si}(\mathrm{Li})$. The photoacoustic signal was detected by a condenser microphone and then amplified by a lock-in amplifier (NF Circuit Block Inc., LI574A with P-51A preamplifier) to be read on a digital voltmeter (HEWLETT PaCKard, 3456A). In the case of a photoacoustic measurement with UV and visible light excitation, the upper beryllium window of the cell was replaced with a quartz window of $1 \mathrm{~mm}$ thickness; the light beam from 


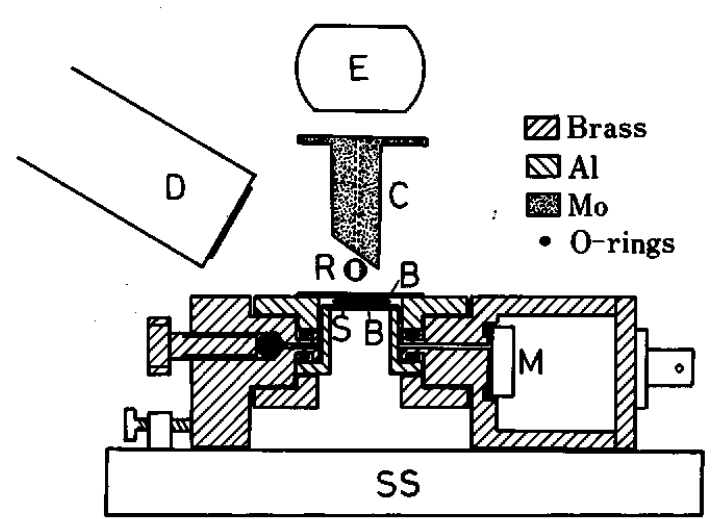

Fig. 1 Schematic diagram of the experimental arrangement for a simultaneous measurement of X-ray photoacoustic and fluorescence, and a sectional view of the photoacoustic cell used for this purpose. S, sample; B, Be window; M, microphone; D, Si(Li) detector; E, Mo target X-ray tube; C, $1 \mathrm{~mm}$ collimator; $\mathrm{R}$, rotating rod chopper; SS, sliding stage.

a $500 \mathrm{~W}$ xenon arc lamp was filtered and focused to a spot of about $5 \mathrm{~mm}$ diameter on the specimen. For UV light excitation, a UV transmisson filter (ToshiBA, UVD-33B) and an IR cut filter (ToshiBA, IRA-25S) were used. In the visible region a UV cut filter (ToshiBA, Y44) and an IR cut filter (ToshIBA, IRA-25S) were used. Sheets of copper foils (thickness $10 \mu \mathrm{m}$ to $1 \mathrm{~mm}$ and diameter 10 to $18 \mathrm{~mm}$ ), laminated sheets of copper foils (thickness $100 \mu \mathrm{m}$, diameter $18 \mathrm{~mm}$ ) and adhesive tapes (thickness about $50 \mu \mathrm{m}$ ), and laminated sheets of lead foils (thickness $100 \mu \mathrm{m}$, diameter $18 \mathrm{~mm}$ ) and aluminum foils (thickness about $15 \mu \mathrm{m}$, diameter $18 \mathrm{~mm}$ ) were used as samples. Copper foils were polished and stored in ethanol. In the case of laminated specimens, polymer adhesive tape or aluminum foil was adhered to both sides of the copper or lead foil.

\section{Results and Discussion}

Photoacoustic and fluorescence signals were simultaneously detected under the conditions of $20 \mathrm{~mA}$ and $40 \mathrm{kV}$ in the X-ray tube. The fluorescence signal, however, was very strong and the dead time of the $\mathrm{Si}(\mathrm{Li})$ detector approached about $90 \%$ under these conditions; thus, the X-ray tube current was reduced to $2 \mathrm{~mA}$ in a measurement of only the fluorescence signal. Figure 2 shows the change in the X-ray photoacoustic and fluorescence $\left(\mathrm{Cu} \mathrm{K}_{\alpha}\right)$ signal intensity plotted as a function of the thickness of the copper foil. Visible photoacoustic signal intensities for the same specimens are also plotted in Fig. 2. The chopping frequency was $8 \mathrm{~Hz}$ for both measurements. The fluorescence signal showed a constant intensity, except for a $10 \mu \mathrm{m}$ thick specimen. Since the critical thickness for X-ray fluorescence is about $30 \mu \mathrm{m}$ for copper $^{7}$ (excitation Mo $K_{\alpha}$, incidental angle $90^{\circ}$, detection angle $30^{\circ}$ ), the fluorescence signal is considered to be independent of

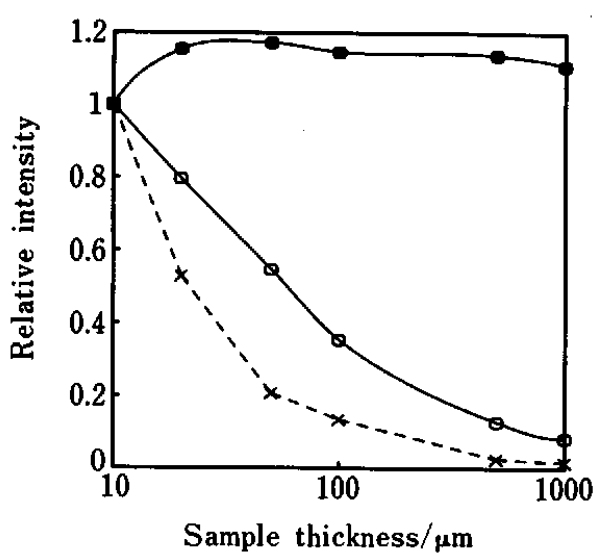

Fig. 2 Photoacoustic and fluorescence signal intensities for different thicknesses of copper foil. (O), X-ray photoacoustic signal; $(X)$, visible photoacoustic signal; $(O)$, X-ray fluorescence signal.

the thickness in the range thicker than $30 \mu \mathrm{m}$. On the other hand, both the X-ray and visible photoacoustic signal intensity depend on the thickness of the specimen. The reason for the decrease in the photoacoustic intensity with an increase in the thickness is considered to be due to heat diffusion in the specimen. The thermal diffusion length for copper at a chopping frequency of $8 \mathrm{~Hz}$ was about $2.2 \mathrm{~mm}$, longer than the thickness of the specimen. Thus, the heat generated by X-ray absorption was considered to be uniformly spread throughout the specimen. Under these conditions, the $\mathrm{X}$-ray photoacoustic signal intensity, $P$, for copper foil of thickness, $t$, was estimated using:

$$
I(E)=k I_{0}(E)[1-\exp (-t \mu(E) \rho)] / t
$$

and

$$
P=\Sigma I(E)
$$

where $I(E), k, I_{0}(E), \mu(E), \rho$ and $E$ are the photoacoustic signal intensity, constant for apparatus, initial X-ray beam intensity, mass absorption coefficient of the specimen, density of the specimen and photon energy of the source beam, respectively. The mass absorption coefficient, $\mu$, is generally given by: ${ }^{8}$

$$
\mu(E)=\tau(E)+\sigma(E)
$$

and

$$
\tau(E)=C E^{-3}-D E^{-4},
$$

where $\tau(E)$ and $\sigma(E)$ are the photoelectric absorption and the scattering loss of the X-ray beam. In photoacoustic measurements only $\tau$ contributes to the generation of a photoacoustic signal. Parameters $C$ and $D$ are functions of the atomic number. Figure 3 shows a log-log plot of the photoacoustic signal 


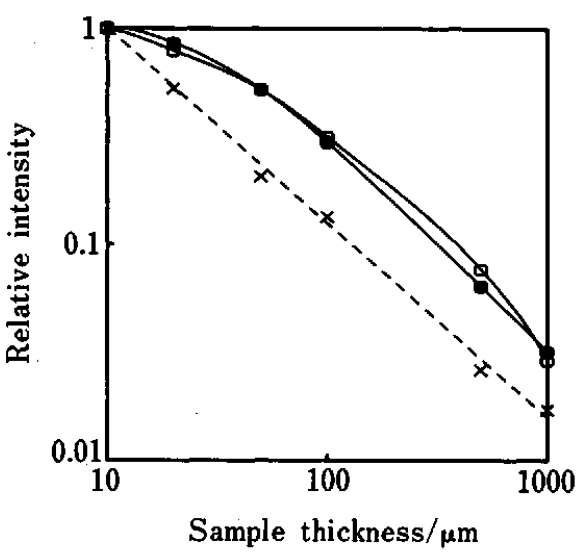

Fig. 3 Log-log plots of photoacoustic and fluorescence signal intensities for different thicknesses of copper foils. Photoacoustic signal intensities estimated from the mass absorption coefficient and the heat capacity are also plotted. (O), X-ray photoacoustic signal; $(X)$, visible photoacoustic signal; $(\Theta)$, estimated $\mathrm{X}$-ray photoacoustic signal.

intensity versus the thickness of the specimen. Estimated values of the $\mathrm{X}$-ray photoacoustic signal intensity were calculated using Eqs. (1) - (4) with the energy distribution of X-ray source in the range 0.5 to $40.5 \mathrm{keV}$. The energy distribution of the $\mathrm{X}$-ray source was measured by an $\mathrm{Si}: \mathrm{Li}$ detector attached to the X-ray fluorescence apparatus using an acrylic resin plate (thickness $10 \mathrm{~mm}$ ) as a diffuser. The visible photoacoustic signal intensity was found to be inversely proportional to the thickness. Visible light was absorbed at the surface of the specimen and the amount of absorbed energy was independent on the thickness of the specimen. Thus, the temperature change was inversely proportional to the heat capacity which, in turn, was proportional to the thickness of the specimen. This supports one of the assumptions that the heat generated in a specimen is spread uniformly within the specimen. The X-ray photoacoustic signal intensities for thin specimens showed a tendency to approach a constant value, as is in agreement with a curve for the calculated values. On the other hand, a decrease in the X-ray photoacoustic signal intensities for thick specimens were found to be smaller than the estimated values. The discrepancy between the experimental value and the estimated value was small and is interpreted in terms of the heat loss by fluorescence, and by the background signal caused by scattered X-rays. Since the intensity of the X-ray photoacoustic signal depends on the thickness of the specimen, its thickness can be estimated if the component of the sample is known or the calibration curve was previously obtained.

When absorption of the exciting beam occurred at the inner part of the sample, the photoacoustic signal showed a phase lag which depended on the thickness of the coating layer and the chopping frequency. This is because any heat generated under the coating is conducted through the coating layer, and is converted

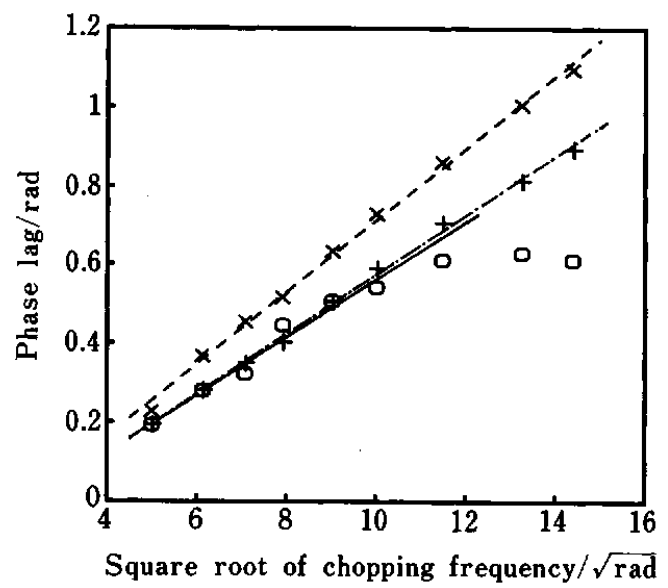

Fig. 4 Phase lag of the photoacoustic signal vs. the square root of the angular chopping frequency for a laminated specimen of copper foil and polymer film. $(O)$, X-ray photoacoustic signal; $(X)$, visible photoacoustic signal; $(+)$, UV photoacoustic signal.

into a photoacoustic signal at the surface. ${ }^{1}$ The following equations give the phase lag, $\phi$ :

$$
\phi=t(2 \alpha)^{-1 / 2} \omega^{1 / 2}+C
$$

and

$$
\alpha=k /(c \rho)
$$

where $\phi, t, \alpha, \omega, C, k, \rho$ and $c$ are the phase lag, the sample thickness, the thermal diffusivity of the sample, the angular chopping frequency, a constant, the thermal conductivity of the sample, the density of the sample, and the heat capacity of the sample, respectively. Figure 4 shows the phase lag of the photoacoustic signal for a laminated sample of copper foil and polymer sheets for various excitation sources. The phase lag showed a linear relation with the square root of the chopping frequency. The polymer layer was transparent for all excitation beams used in this experiment. The slopes of the regression line determined by a least-square fitting were $0.073,0.076$ and $0.092 \mathrm{~s}^{1 / 2}$ for $\mathrm{X}$-ray, UV and visible light excitation, respectively. The density of the polymer layer was $1.33 \mathrm{~g} / \mathrm{cm}^{3}$. If the heat capacity of the polymer layer was known, the thermal conductivity could be calculated. Assuming that the heat capacity of the polymer layer was about $2 \mathrm{~J} \mathrm{~g}^{-1} \mathrm{~K}^{-1}$, the thermal conductivity of the polymer layer was calculated to be about $3.9 \times 10^{-3}$, $5.8 \times 10^{-3}$ and $6.2 \times 10^{-3} \mathrm{~J} \mathrm{~cm}^{-1} \mathrm{~s}^{-1} \mathrm{~K}^{-1}$, respectively. Since the polymer layer absorbed UV and X-rays, the heat generated by absorbing the beam near the surface would make the phase lag small for UV and X-ray excitation. On the other hand, the phase lag for a laminated specimen of lead foil and aluminum foil (Fig. 5) shows a somewhat different behavior. Since aluminum is transparent for $\mathrm{X}$-rays, the phase lag 


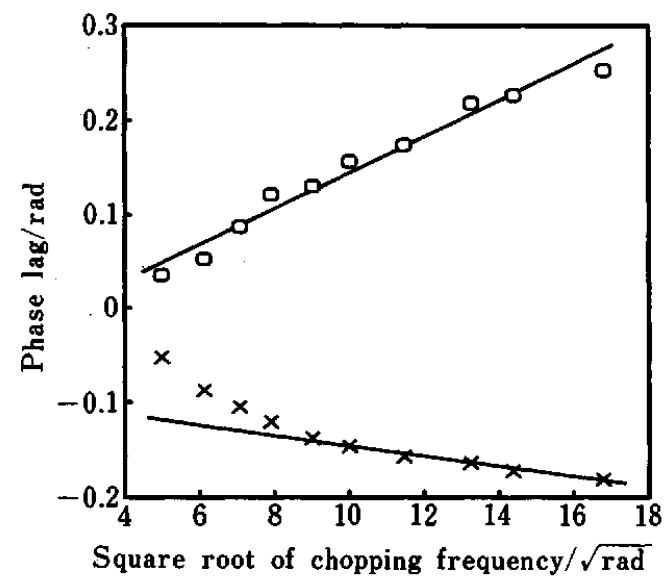

Fig. 5 Phase lag of the photoacoustic signal vs. the square root of the angular chopping frequency for a laminated specimen of lead foil and aluminum foil. $(O), X$-ray photoacoustic signal; $(X)$, visible photoacoustic signal.

increased proportionally to the square root of the chopping frequency. On the other hand, aluminum is opaque to visible light; thus, the phase lag is constant for the chopping frequency. The slope of the regression line determined by least-square fitting for an X-ray photoacoustic signal was $0.019 \mathrm{~s}^{1 / 2}$. This value is large compared to the estimated value $\left(1.2 \times 10^{-3} \mathrm{~s}^{1 / 2}\right)$ from the thickness $(15 \mu \mathrm{m})$, thermal conductivity $\left(2.04 \mathrm{~J} \mathrm{~cm}^{-1}\right.$ $\left.\mathrm{s}^{-1} \mathrm{~K}^{-1}\right)$, density $\left(2.69 \mathrm{~g} / \mathrm{cm}^{3}\right)$ and heat capacity $\left(0.895 \mathrm{~J} \mathrm{~g}^{-1}\right.$ $\mathrm{K}^{-1}$ ) of the aluminum layer. The adhesive layer between the aluminum and lead is considered to be the reason for the phase lag. Assuming that the thermal conductivity, density and heat capacity of the adhesive layer were equal to those of poly-methacrylate $\operatorname{resin}^{9}$ $\left(1.5 \times 10^{-3} \mathrm{~J} \mathrm{~cm}^{-1} \mathrm{~s}^{-1} \mathrm{~K}^{-1}, \quad 1.2 \mathrm{~g} / \mathrm{cm}^{3}\right.$ and $\left.1.4 \mathrm{~J} \mathrm{~g}^{-1} \mathrm{~K}^{-1}\right)$, the thickness of the layer was estimated to be about 8 $\mu \mathrm{m}$ from the slope $\left(0.019 \mathrm{~s}^{1 / 2}\right)$. This value was comparable to that determined by microphotographic observations. Information concerning the property of surface layer can, thus, be obtained from the relation between the phase lag and the chopping frequency.

Figure 6 shows the simultaneously measured photoacoustic and fluorescence $\left(\mathrm{Pb} \mathrm{L}_{\alpha}\right)$ signal change of the specimen. The fluorescence signal shows the presence of the lead layer. The X-ray and visible photoacoustic signals indicate that the X-ray absorption coefficient of the inner layer at the center part of the specimen is low, and that the heat conductivity of the inner layer is lower than that for lead. The phase lag of the photoacoustic signal by visible light excitation showed a small deviation. This indicates that the absorption of visible light occurred at the surface layer of the specimen. On the other hand, the phase lag by X-ray excitation showed a large deviation, which indicates a change in the average depth where $\mathrm{X}$-ray absorption occurred.
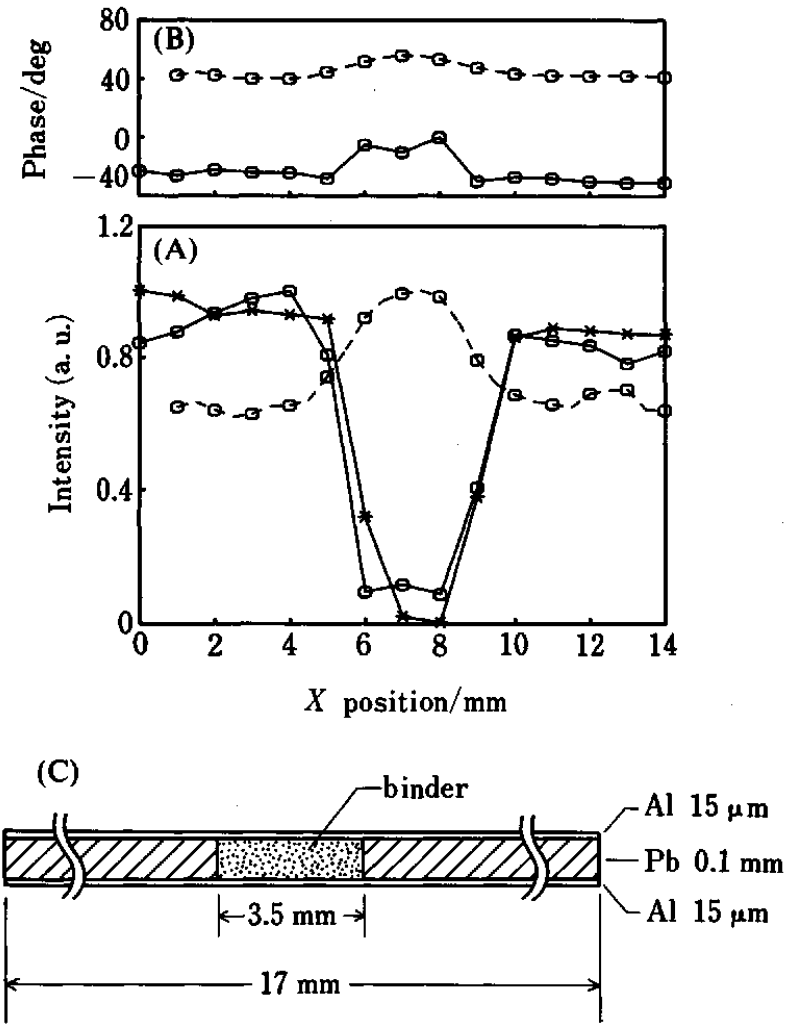

Fig. 6 Simultaneously measured photoacoustic and fluorescence signals (A) by $X$-ray excitation for a model laminated specimen (C). The photoacoustic signal for the same specimen upon visible light excitation is also plotted. Upper traces (B) show the phase lags of photoacoustic signals. $(\theta)$, $X$-ray photoacoustic signal; $(\Theta)$, visible photoacoustic signal; (*), X-ray fluorescence signal.

\section{References}

1. Rosencwaig, "Photoacoustics and Photoacoustic Spectroscopy", John Wiley \& Sons, New York, 1980.

2. S. Mascarennas, H. Vargas and C. L. Cesar, Med. Phys., 11, 73 (1984).

3. R. Germer, Rev. Sci. Instrum., 55, 1461 (1984).

4. K. Kato, Y. Kobayashi, S. Aoki and Y. Sugitani, Anal. Sci., 3, 275 (1987).

5. T. Masujima, H. Kawata, Y. Amemiya, N. Kamiya, T. Katsura, T. Iwamoto, H. Yoshida, H. Imai and M. Ando, Chem. Lett., 1987, 937.

6. K. Kato and Y. Sugitani, Anal. Sci, 4, 59 (1988).

7. K. Ohno, A. Kawase and T. Nakamura, "Ekkususen Bunsekiho (X-ray Analysis, in Japanese)", p. 22, Kyoritsushuppan, Tokyo, 1987.

8. C. H. Macgillary, G. D. Rieck and K. Lonsdale, "International Tables for X-ray Crystallography", Vol. 3, p. 175, Kynoch Press, Birmingham, 1962.

9. "Kagaku-binran Kisohen II (Handbook of Chemistry, in Japanese)", ed. Nihonkagakukai, 2nd ed., pp. 902, 986, Maruzen, Tokyo, 1975. 